\title{
Routine stair climbing for vascular health
}

\author{
Hirofumi Tomiyama ${ }^{1}$
}

Received: 8 June 2021 / Revised: 15 June 2021 / Accepted: 21 June 2021 / Published online: 19 July 2021

(C) The Japanese Society of Hypertension 2021

Atherosclerotic vascular damage plays a key role in the development of cardiovascular disease (CVD) [1, 2]. Therefore, the management of atherosclerotic vascular damage is crucial (i.e., maintaining vascular health) $[1,2]$. In addition to the management of conventional risk factors for CVD, lifestyle modification is also recommended [3, 4]. Increased physical activity is beneficial for conventional risk factors for cardiovascular disease such as hypertension, glucose/lipid metabolism, and/or obesity, and maintaining good physical activity may maintain vascular health [3, 4]. In Japan, the "Physical Activity Criteria for Health 2013" recommends that among individuals with health checkup data within the criterion range, those aged 65 years or older should perform physical activity of any intensity for $40 \mathrm{~min}$ or more every day and those aged 18-64 years should perform physical activity at an intensity of 3 Mets or higher for $60 \mathrm{~min}$ or more every day [5].

However, methods to assess physical activity have not been fully established. Questionnaires are one of the methods, but they take time (Fig. 1). In clinical practice, for the management of patients with hypertension, a simpler method is needed. In this issue, Yamaji et al. proposed a simple question: "Up to how many flights of stairs do you usually use for climbing?" is a simple alternative approach to identify patients who participate in physical activity that is good for vascular health (Fig. 1) [6]. This question does not reflect the extent of physical activity. However, the flowmediated vasodilatation of the brachial artery (FMD) in the patients answered "over the third floor" was higher than that in the patients answered "the second floor or none". Endothelial dysfunction is an initial step of atherosclerotic vascular damage, and FMD is a marker of endothelial function $[7,8]$. A recent meta-analysis conducted by Matsuzawa et al.

Hirofumi Tomiyama

tomiyama@tokyo-med.ac.jp

1 Department of Cardiology, Tokyo Medical University, Tokyo, Japan reported that FMD significantly predicted cardiovascular events (adjusted relative risk [95\% CI]: 1\% increase in FMD 0.88 [0.84-0.91]) [9]. Thus, it is possible that the physical activity in the patients who answered "over the third floor" provides better cardiovascular outcomes than that in patients who answered "the second floor or none".

The FMD-J study is a multicenter prospective observational study conducted in Japan (i.e., all study participants were Japanese men and women) to examine the usefulness of FMD to predict the development of subclinical organ damage or new-onset cardiovascular events [10]. In a subanalysis of the FMD-J study, Maruhashi et al. reported that FMD above the cutoff value of $7.1 \%$, derived from receiveroperator curve analyses for cardiovascular outcomes, was significantly associated with a lower risk of the outcomes [11]. In addition, in another subanalysis of FMD-J studies, the median FMD was 7.2\% (interquartile range, 5.2\%-9.1\%) in subjects aged 30 to 74 years without cardiovascular risk factors or cardiovascular disease [12]. Based on these findings, we proposed that the cutoff value for normal endothelial function is $7 \%$ [12]. In Yamaji's study, the FMD value in patients who answered "over the third floor" was 3-4\% [6]. This value was low compared to the normal range of FMD [10, 12]. As mentioned above, Matsuzawa et al. proposed a linear relationship of FMD value with cardiovascular events. Therefore, further study is needed to clarify whether low FMD values provide favorable cardiovascular outcomes compared with even lower FMD values.

Yamaji et al. could not determine the mechanisms underlying the significant difference in FMD between patients who answered "over the third floor" and those who answered "the second floor or none". One possibility might be that this difference reflects the difference in the commitment to maintaining physical activity. Maintaining a good level of physical activity is an important issue [13]. Many environmental factors affect adherence. For example, the COVID-19 pandemic lockdown had a large negative impact on maintaining a healthy lifestyle [14]. Physical and mental stresses in daily life and/or job strain may disturb the 
Fig. 1 Approaches to assess physical activity in patients

\section{Conventional approach}

Information on physical activity was obtained by using the self-reported Physical Activity Questionnaire. This questionnaire consists of 4 questions on

1. Exercise habit

2. Frequency of each intensity of exercise for more than 10 minutes each time (times/week) (light intensity, moderate intensity, and vigorous intensity)

3. Duration of each exercise time (minutes)

4. Sitting time

\section{Simple approach}

Up to how many flights of stairs do you usually use for climbing?

Answer: Climbing to over $3^{\text {rd }}$ floor.

Possibly, you may have a good physical activity for vascular health

maintenance of a good level of physical activity. Therefore, it is necessary to clarify whether the patients who answered "over the third floor" have a strong will to maintain good physical activity.

\section{Compliance with ethical standards}

Conflict of interest The author declares no competing interests.

Publisher's note Springer Nature remains neutral with regard to jurisdictional claims in published maps and institutional affiliations.

\section{References}

1. Arnold N, Lechner K, Waldeyer C, Shapiro MD, Koenig W. Inflammation and cardiovascular disease: the future. Eur Cardiol. 2021;16:e20.

2. Soehnlein O, Libby P Targeting inflammation in atherosclerosisfrom experimental insights to the clinic. Nat Rev Drug Discov. $2021 ; 11 ; 1-22$.

3. Man AWC, Li H, Xia N. Impact of lifestyles (diet and exercise) on vascular health: oxidative stress and endothelial function. Oxid Med Cell Longev. 2020;2020:1496462.

4. Arnett DK, Blumenthal RS, Albert MA, Buroker AB, Goldberger ZD, Hahn EJ, et al. 2019 ACC/AHA Guideline on the Primary Prevention of Cardiovascular Disease: A Report of the American College of Cardiology/American Heart Association Task Force on Clinical Practice Guidelines. Circulation. 2019; 140:e596-e646.

5. Physical Activity Criteria for Health 2013 https://www.e-hea lthnet.mhlw.go.jp/information/exercise/s-01-001.html.
6. Yamaji T, Harada T, Hashimoto Y, Nakano Y, Kajikawa M, Yoshimura K, et al., Stair climbing activity and vascular function in patients with hypertension. Hypertens Res. https://doi.org/10. 1038/s41440-021-00697-z.

7. Tomiyama H, Yamashina A. Non-invasive vascular function tests: their pathophysiological background and clinical application. Circ J. 2010;74:24-33.

8. Higashi Y, Kihara Y, Noma K. Endothelial dysfunction and hypertension in aging. Hypertens Res. 2012;35:1039-1047.

9. Matsuzawa Y, Kwon TG, Lennon RJ, Lerman LO, Lerman A. Prognostic value of flow-mediated vasodilation in brachial artery and fingertip artery for cardiovascular events: a systematic review and meta-analysis. J Am Heart Assoc. 2015; 4:e002270.

10. Tomiyama H, Kohro T, Higashi Y, Takase B, Suzuki T, Ishizu T, et al. Reliability of measurement of endothelial function across multiple institutions and establishment of reference values in Japanese. Atherosclerosis. 2015;242:433-442.

11. Maruhashi T, Soga J, Fujimura N, Idei N, Mikami S, Iwamoto Y, et al. Endothelial dysfunction, increased arterial stiffness, and cardiovascular risk prediction in patients with coronary artery disease: FMD-J (Flow-Mediated Dilation Japan) Study A. J Am Heart Assoc. 2018;7:e008588.

12. Tanaka A, Tomiyama H, Maruhashi T, Matsuzawa Y, Miyoshi T, Kabutoya T, et al. Physiological diagnostic criteria for vascular failure. Hypertension. 2018;72:1060-1071.

13. Tam HL, Wong EML, Cheung K. Effectiveness of educational interventions on adherence to lifestyle modifications among hypertensive patients: an integrative review. Int $\mathrm{J}$ Environ Res Public Health. 2020;17:2513.

14. Nguyen MH, Pham TTM, Nguyen KT, Nguyen YH, Tran TV, Do BN, et al. Negative impact of fear of COVID-19 on health-related quality of life was modified by health literacy, ehealth literacy, and digital healthy diet literacy: a multi-hospital survey. Int $\mathbf{J}$ Environ Res Public Health. 2021;18:4929. 